\title{
Attitudes toward the use of humanoid robots in healthcare-a cross-sectional study
}

\author{
Malin Andtfolk ${ }^{1} \oplus \cdot$ Linda Nyholm $^{1} \cdot$ Hilde Eide $^{2} \cdot$ Auvo Rauhala $^{1} \cdot$ Lisbeth Fagerström $^{1,2}$
}

Received: 6 October 2020 / Accepted: 23 August 2021 / Published online: 4 September 2021

(c) The Author(s) 2021

\begin{abstract}
The use of robotic technology in healthcare is increasing. The aim was to explore attitudes toward the use of humanoid robots in healthcare among patients, relatives, care professionals, school actors and other relevant actors in healthcare and to analyze the associations between participants' background variables and attitudes. The data were collected through a cross-sectional survey $(N=264)$ in 2018 where participants met a humanoid robot. The survey was comprised of background variables and items from a modified Robot Attitude Scale. Multiple linear regression analysis and Spearman's Rho correlation were used to analyze associations between variables. Most of the participants were positive toward the use of humanoid robots in healthcare and only a few were negative. Attitudes toward the use of humanoid robots were more positive among other relevant actors, such as service personnel and politicians in healthcare, participants with a higher educational level and older adults. More research is needed on the reasons underlying negative attitudes because these might affect the introduction of humanoid robots in healthcare. A careful evaluation of appropriate first target groups as well as which tasks are appropriate for humanoid robots to perform in healthcare are needed.
\end{abstract}

Keywords Humanoid robot $\cdot$ Attitudes $\cdot$ Health care $\cdot$ Cross-sectional $\cdot$ Logistic regression

\section{Abbreviation \\ RAS Robot Attitude Scale}

\section{Introduction}

As an answer to the rising workload and costs in healthcare, use of robot technology is increasing in the field (Dahl and Boulos 2013). Robot technology looks promising in meeting challenges such as the increase of the aging population and the decrease in number of care professionals (McGeeLennon et al. 2011; Zsiga et al. 2013; Baer et al. 2014). To gain a deeper understanding of the use of robot technology in healthcare, we investigated attitudes toward a specific robot solution: humanoid robots, which are robots designed

Malin Andtfolk

malin.andtfolk@abo.fi

1 Faculty of Education and Welfare Studies, Department of Caring Science, Åbo Akademi University, PO Box 311, FI-65101 Vaasa, Finland

2 Faculty of Health and Social Sciences, Department of Nursing Science, University of South-Eastern Norway, Drammen, Norway to look, behave or interact in a "human-like" manner (Azeta et al. 2018; Kyrarini et al. 2021; Winfield 2012) as well as perform basic human movements (Brunda et al. 2020). With their multi-flexible functions, humanoid robots have been used to, e.g., assist patients with reminders about medication (Doering et al. 2015), educate about diet adherence or walk with patients (Ishiguro et al. 2016). The use of humanoid robots in healthcare is a much-contested phenomenon, with mixed attitudes and acceptance seen in earlier studies. To date, there are only a few studies specifically investigating attitudes toward humanoid robots. Earlier studies (Pripfl et al. 2016; Papadopoulus et al. 2018) have found that both patients and care professionals generally accept and understand the benefits of using humanoid robots. Pripfl et al. (2016) investigated challenges such as errors in humanoid robots' functions that could lead to frustration and whether usability was negatively influenced by humanoid robots' lack of robustness. Papadopoulus et al. (2018) reported that care professionals were concerned with issues such as patient safety and privacy in healthcare when using humanoid robots or animal-like robots.

Individual and cultural differences such as gender (Kuo et al. 2009) or earlier experiences with robots (Heerink 2011) might play a considerable role in attitudes toward 
robots. Kuo et al. (2009) found that younger adults, men and those with a higher educational level showed a more positive attitude toward social assistive robots than older persons, women and those with a lower education. In other studies, patients were more likely to have a more positive attitude toward healthcare robots than care professionals (Broadbent et al. 2012) and showed a significantly more positive attitude toward such robots (Broadbent et al. 2010). In another study (Giusti and Marti 2006), robot-related features were seen to possibly positively influence older persons' attitudes toward accepting technology. Alaid and Zhou (2014) found that nurses appreciated using home healthcare robots in tasks that required social interaction but that they also expressed ethical and privacy concerns with using robots. In another study, nurses were found to not support the use of humanoid robots in healthcare (Ito et al. 2015). None of the aforementioned studies included a focus on humanoid robots, and overall only a few studies have included such a specific focus. Some studies have included robot prototypes (Nomura et al. 2012, 2015), while in others the robots were not tested in real-life situations (Heerink 2011; Alaid and Zhou 2014). Given that the development and implementation of humanoid robots in healthcare is advancing, it is of interest to study a broader scope of attitudes toward humanoid robots, including the background variables that might affect attitudes, either positively or negatively. In this study, we sought to reveal the range of attitudes that may exist.

\section{Aim and research questions}

The aim of this study was to explore attitudes toward the use of humanoid robots in healthcare among patients, relatives, care professionals, school actors and other relevant actors in the healthcare field and to analyze the associations between participants' background variables and attitudes. The research questions were: (1) What are the attitudes toward the use of humanoid robots in healthcare, and (2) What are the associations between participants' background variables and their attitudes?

\section{Material and methods}

\subsection{Study design and setting}

This study had a cross-sectional descriptive design and was conducted in Finland in November and December 2018. It was performed in compliance with the ethical principles delineated by the Finnish National Board on Research Integrity (2002). To include a broader perspective in the study, we recruited persons visiting or working at a hospital, such as; patients, relatives, care professionals, school actors and other relevant actors in the healthcare field (i.e. service personnel and politicians). We also recruited conference delegates visiting a caring conference, which included care professionals and other relevant actors in healthcare. A notice about the study, including information about the study, was placed on the website of the hospital included in the study and in a newsletter for the conference. The recruitment consisted of the random selection of participants, where all working at or visiting the hospital, or attending the conference were given the opportunity to participate during the time of data collection. The study sample consisted of 264 participants.

The Pepper humanoid robot was used in this study; it can be used for interaction, rehabilitation and to support everyday-life (SoftBank Robotics 2017). During data collection, the humanoid robot was stationed in a lobby at either the hospital or the conference center, with the aim to provide interaction. Depending on how much time the participants had (e.g., appointment with a care professional, lunchbreak), each participant met the humanoid robot for 15-30 min. The participants were asked to actively interact with the humanoid robot, both alone and in small groups. The participants were given a list of suggested questions and sentences, which facilitated interaction with the humanoid robot. The humanoid robot had the following functionalities: greeting the participants in three languages (Finnish, Swedish or English), answering basic questions about time, weather, etc., playing music and interactive games, performing dances and singing. Furthermore, the humanoid robot interacted with the participants by walking toward them and initiating a discussion. The function of the humanoid robot depended on both its status as well as the participants' own preferences. All participants were asked to fill in a paper survey (conducted in either Finnish, Swedish or English) on a voluntary basis, immediately after the interaction.

\subsection{Measures}

\subsubsection{The Robot Attitude Scale}

The paper survey was comprised of items from the Robot Attitude Scale (RAS) and background variables, i.e., sociodemographic variables and information about earlier experiences with humanoid robots. Developed by Broadbent et al. (2009), the RAS is used to measure attitudes toward robots and has been investigated in previous healthcare settings (Broadbent et al. 2009, 2010, 2012; Stafford et al. 2014). The RAS was used here to investigate participants' attitudes toward the use of humanoid robots in healthcare. The original RAS consists of 11 items where positive and negative assumptions are weighed against one another, such as friendly-unfriendly, useful-useless, trustworthy-untrustworthy, strong-fragile, interesting-boring, advanced-basic, easy to use-hard to use, reliable-unreliable, safe-dangerous, 
simple-complicated, and helpful-unhelpful, with scores from 1 to 8 . A low score indicates a more positive attitude, while a high score indicates a more negative attitude. After discussion with the original developers of the scale (Broadbent et al. 2009), approval was given to modify the original RAS with the aim to minimize participant burden. All 11 items from the original RAS were included in the modified version used here, called the RAS-5, but the scores were shortened from $1-8$ to $1-5$. That is to say, the original RAS included, e.g., the item friendly-unfriendly with scores from 1 to 8 , while the modified RAS-5 also included the item friendly-unfriendly but with scores from 1 to 5 . A participant's RAS-5 score was calculated as an average of all his/ her ratings (1-5) for all individual items. Permission was also granted for linguistic modification of the instrument; the original RAS was translated from the English language into both the Swedish and Finnish languages.

To achieve equivalence between the various language versions of the instrument (Sperber 2004), the guidelines for the Process of Cross-Cultural Adaption and validation by Beaton et al. (2000) were used. The validation process included six stages: translation, synthesis, back translation, expert committee review, pretesting, submission and appraisal of all written reports (Table 2). In stage one, four translators were used to translate the instrument into the target languages: two whose first language is the Swedish language, two whose first language is the Finnish language. These translators each produced a written report of the completed translation. Per language, one translator was aware of the study concept (informed), while the other was not (uninformed). In stage two, all four translators worked with the study's first author (NN) to synthesize the translation results. Together, one common translation per language was produced (T-12, T-34). In stage three, two translators whose first language is the English language performed backtranslations; neither were aware of the study concept (uninformed). Working from the T-12 and T-34 translations, two back-translations per language were produced (BT1, BT2, BT3, BT4). In stage four, an expert committee composed of four persons reviewed all reports and translations (T1, T2, T3, T4 and BT1, BT2, BT3, BT4) and reached a consensus of synthesis, yielding a pre-final version of the RAS-5 for pretesting. In stage five, pretesting and adaption of the survey occurred, and in stage six the newly translated survey was approved.

A total of 34 persons of various ages and nursing experience tested the RAS- 5 for comprehensibility. Both language versions of the survey were considered to retain equivalence in the applied test situations and, consequently, constitute reasonable translations. Whenever an instrument is translated and used in a new context, its reliability should be tested (Pallant 2011). Cronbach's Alpha was used to test the internal consistency of the RAS-5, estimated as 0.87 (Table 1).

\subsubsection{Background variables}

The background variables were: Participant group (patients, relatives, care professionals, healthcare students, $\mathrm{PhD}$ students or researchers in healthcare, healthcare teachers, other relevant actors in healthcare), Gender (woman/man/ other), Age (15-19, 20-29, 30-39, 40-49, 50-59, 60-69, 70-79, 80+ years), Mother language (Swedish/Finnish/English/Other), Educational level (basic education, vocational school, higher secondary vocational school. high school, university of applied sciences, university), Read/heard (yes/
Table 1 Recommended stages of cross-cultural adaption

\begin{tabular}{|l|}
\hline $\begin{array}{l}\text { Stage I: } \\
\text { Translation }\end{array}$ \\
\hline $\begin{array}{l}\text { Stage II: } \\
\text { Synthesis }\end{array}$ \\
\hline \hline $\begin{array}{l}\text { Stage III: } \\
\text { Back } \\
\text { translation }\end{array}$ \\
\hline $\begin{array}{l}\text { Stage IV: } \\
\text { Expert } \\
\text { committee } \\
\text { review }\end{array}$ \\
\hline $\begin{array}{l}\text { Stage V: } \\
\text { Pretesting }\end{array}$ \\
\hline
\end{tabular}

Two translations from English into Swedish language (T1, T2)

Two translations from English into Finnish language (T3, T4)

One informed + one uninformed translator per language

Synthesize T1 \& T2 into T-12

Synthesize T3 \& T4 into T-34

Resolve discrepancies with translators' reports

Two translators with English as mother language

Work from $\mathrm{T}-12$ and $\mathrm{T}-34$ versions

Create two back-translations per language (BT1, BT2, BT3, BT4)

Review all reports

Reach consensus on synthesis

Create a pre-final version

$\mathrm{N}=34$

Complete survey (pilot-testing with small sample)

Investigate to get understanding of items 
Table 2 Descriptive statistics of other background variables in participant groups

\begin{tabular}{|c|c|c|c|c|c|c|c|}
\hline \multicolumn{2}{|c|}{ Participant group } & \multirow{2}{*}{$\begin{array}{l}\text { Patients } \\
27(10.2 \%)\end{array}$} & \multirow{2}{*}{$\begin{array}{l}\text { Relatives } \\
20(7.6 \%)\end{array}$} & \multirow{2}{*}{$\begin{array}{l}\text { Care professionals** } \\
67(25.4 \%)\end{array}$} & \multirow{2}{*}{$\begin{array}{l}\text { School actors } * * * \\
75(28.4 \%)\end{array}$} & \multirow{2}{*}{$\begin{array}{l}\text { Other**** } \\
75(28.4 \%)\end{array}$} & \multirow{2}{*}{$\begin{array}{l}\text { Total } \\
264(100 \%)\end{array}$} \\
\hline$n^{*}(\%)$ & & & & & & & \\
\hline \multirow[t]{2}{*}{ Gender } & Woman & 14 & 12 & 60 & 70 & 58 & 214 \\
\hline & Man & 13 & 8 & 7 & 5 & 17 & 50 \\
\hline \multirow[t]{8}{*}{ Age } & $15-19$ & - & 1 & - & 2 & 1 & 4 \\
\hline & $20-29$ & 3 & - & 11 & 16 & 7 & 37 \\
\hline & $30-39$ & 1 & 6 & 10 & 25 & 7 & 49 \\
\hline & $40-49$ & 1 & 2 & 19 & 19 & 17 & 58 \\
\hline & $50-59$ & 5 & 2 & 22 & 12 & 32 & 73 \\
\hline & $60-69$ & 6 & 3 & 4 & 1 & 7 & 21 \\
\hline & $70-79$ & 9 & 5 & 1 & - & 3 & 18 \\
\hline & $80-89$ & 2 & 1 & - & - & 1 & 4 \\
\hline \multirow[t]{3}{*}{ Language } & Swedish & 14 & 13 & 41 & 37 & 45 & 150 \\
\hline & Finnish & 12 & 6 & 21 & 31 & 29 & 99 \\
\hline & Other & 1 & 1 & 5 & 7 & 1 & 15 \\
\hline \multirow[t]{2}{*}{ Education } & Lower & 13 & 9 & 13 & 29 & 15 & 79 \\
\hline & Higher & 12 & 10 & 51 & 46 & 60 & 179 \\
\hline \multirow[t]{2}{*}{ Read/heard } & Yes & 13 & 15 & 54 & 69 & 62 & 213 \\
\hline & No & 11 & 4 & 11 & 6 & 12 & 44 \\
\hline \multirow[t]{2}{*}{ Have met } & Yes & 1 & - & 10 & 17 & 15 & 43 \\
\hline & No & 23 & 20 & 55 & 58 & 60 & 216 \\
\hline
\end{tabular}

* $n$ denotes the sub-sample size

**Care professionals including physicians

***Students, $\mathrm{PhD}$ students, researchers and teachers in healthcare

$* * * *$ Other relevant actors in healthcare

no, have you read/heard about humanoid robots before), and Have met (yes/no, have you met a humanoid robot before).

Prior to analyses, some background variables were dichotomized. The participant groups were reclassified as Patients, Relatives, Care professionals (care professionals and physicians became Care professionals, due to similar context), School actors (students, PhD students, researchers, teachers in healthcare became School actors, due to similar context) and Other (other relevant actors in healthcare). Gender was reclassified as woman/man (no gender type other was seen). Age was reclassified as 15-19, 20-29, 30-39, 40-49, 50-59, 60-69, 70-79, 80-89 (no participant over 90 years old was seen). Mother language was reclassified as Swedish/Finnish/Other (English and other were merged into Other due to low amount in each category). Educational level was reclassified as lower/higher (due to small amount in each level; categories from higher secondary vocational school upward were included in higher, with the remainder in lower).

\subsection{Statistical analyses}

Categorical variables were presented as frequencies and percentages. RAS-5 scores were reported with mean, median and standard deviation and in some instances with 95\% confidence intervals. Differences in mean RAS- 5 scores between background variables were analyzed with a $T$ test of independent samples when comparing two classes and with oneway ANOVA when comparing three or more classes. When using one-way ANOVA to compare the means of RAS-5 items, post-hoc comparisons were also performed with the Scheffe test. To analyze the association between background variables and RAS-5 score, multiple linear regression analysis was used. Age was an ordered scale variable with all eight classes one decade in size, except for the first class which was 15-19 years. Accordingly, age was treated as a quantitative variable, with a unit of one decade. The assumptions of multiple linear regression were tested and fulfilled: the variance of residuals was homogenous, their distribution was normal and there was no correlation between them, and there was no significant multicollinearity. However, the relationship between the RAS-5 score and age was not completely linear. Preliminary analysis was also performed with ANCOVA, using identical variables as in multiple linear regression analysis to test all pairwise interactions. None were found to be statistically significant. Consequently, a multiple linear regression analysis method was used for all further regression analyses. The association between the 
background variables and dichotomized RAS-5 was also analyzed with Spearman's Rho correlation analysis. All the variables for the category Participant group were dummy coded. The variable age was dichotomized into groups of $15-49$ and 50 or over, due to equal numbers of participants in each group. Accordingly, all background variables were dichotomous in the correlation analysis. The mean RAS-5 score of 2.3806 was chosen as the cut-off level for dichotomization. Two-tailed analyses were conducted and statistical significance was set at $P$ values below 0.05 . All data were analyzed with SPSS Statistics version 25 (IBM Corporation, Armonk, NY, USA).

\section{Results}

\subsection{Participant characteristics}

An overall description of participants' background variables is presented in Table 2. There was a total of 264 participants, and the clear majority were women (81\%). Most were 15-49 years of age (56\%), had Swedish as their mother language (57\%), had a higher education (69\%), or had read/ heard about humanoid robots before (83\%). Only a few had met a humanoid robot before (17\%).

\subsection{Overall RAS-5 results}

Total frequencies (\%) of the 11 RAS-5 items with scores from 1 to 5 were: score 1, 613 ratings (21.3\%); score 2, 940 ratings (32.7\%); score 3, 997 ratings (34.7\%); score 4, 257 ratings (8.9\%); score 5, 66 ratings (2.3\%). This indicates that most of the participants had mainly positive attitudes toward most RAS-5 items and thus the use of humanoid robots in healthcare. The one-way ANOVA and post-hoc comparisons with the Scheffe test indicated that the mean score of individual RAS-5 items did not differ significantly between items, presented in Table 3. Nevertheless, the items Interesting-boring and Friendly-unfriendly had the lowest mean scores, indicating that the participants had a rather positive attitude toward the humanoid robot and considered it to be interesting and friendly. The item Interesting had a mean score of 1.72 (95\% CI, 1.61 to 1.84 ) and the item Friendly a mean score of 1.92 (95\% CI, 1.81 to 2.04). All other items' mean scores were above these two items' $95 \%$ CI.

\subsection{RAS-5 score according to background variables}

RAS-5 scores of background variables are presented in Table 4. For the variable Participant group, patients had a higher score. For Gender, women had a higher score. For Age, the 30-39 age class had a higher score. For Mother language, those with Finnish as their mother language had a higher score. For Educational level, those with a lower educational level had a higher score. With regard to earlier experiences, in Read/heard and Have met, those who previously had not done either of those things had a higher score. Higher scores suggest a more negative attitude toward the use of humanoid robots in healthcare. For the Spearman's rank correlation between dichotomous RAS-5 and background variables, when compared to all dummy coded participants, other relevant actors in healthcare $(r=-0.211$,

Table 3 Mean scores (SD) for individual RAS-5 items, by participant group

\begin{tabular}{|c|c|c|c|c|c|c|}
\hline $\begin{array}{l}\text { Participant group } \\
(n=264)\end{array}$ & Patients & Relatives & Care professionals & School actors* & Other** & $\begin{array}{l}\text { Mean RAS-5 } \\
\text { score for all } \\
\text { items }\end{array}$ \\
\hline Friendly-unfriendly & $2.30(1.03)$ & $1.75(0.78)$ & $1.91(0.95)$ & $2.08(1.02)$ & $1.69(0.87)$ & $1.92(0.96)$ \\
\hline Useful-useless & $2.85(1.23)$ & $1.90(1.07)$ & $2.35(1.03)$ & $2.37(0.93)$ & $1.99(0.79)$ & $2.27(0.99)$ \\
\hline Trustworthy-untrustworthy & $2.78(1.28)$ & $2.53(0.84)$ & $2.65(0.90)$ & $2.76(0.93)$ & $2.41(0.81)$ & $2.62(0.93)$ \\
\hline Strong-fragile & $2.91(1.24)$ & $2.58(1.07)$ & $2.41(0.99)$ & $2.80(0.89)$ & $2.42(0.85)$ & $2.59(0.97)$ \\
\hline Interesting-boring & $2.11(1.31)$ & $1.85(1.18)$ & $1.66(0.83)$ & $1.79(0.98)$ & $1.55(0.81)$ & $1.72(0.96)$ \\
\hline Advanced-basic & $2.37(1.27)$ & $2.20(1.11)$ & $2.31(0.94)$ & $2.68(0.84)$ & $2.32(0.89)$ & $2.42(0.96)$ \\
\hline Easy to use-hard to use & $2.83(1.07)$ & $2.42(0.84)$ & $2.80(0.92)$ & $2.88(0.84)$ & $2.50(0.88)$ & $2.71(0.90)$ \\
\hline Reliable-unreliable & $2.63(1.24)$ & $2.58(0.90)$ & $2.70(0.78)$ & $2.77(0.85)$ & $2.43(0.79)$ & $2.63(0.87)$ \\
\hline Safe-dangerous & $2.41(1.12)$ & $2.16(0.96)$ & $2.50(0.77)$ & $2.45(0.69)$ & $2.47(0.71)$ & $2.44(0.79)$ \\
\hline Simple-complicated & $3.08(1.29)$ & $2.63(1.12)$ & $2.55(1.01)$ & $2.91(0.96)$ & $2.56(0.92)$ & $2.71(1.00)$ \\
\hline Helpful-unhelpful & $2.48(1.31)$ & $2.11(0.94)$ & $2.27(0.95)$ & $2.29(0.94)$ & $1.92(0.82)$ & $2.19(0.97)$ \\
\hline
\end{tabular}

SD Standard deviation

RAS- 5 scores: $1=$ totally agree, $2=$ agree, $3=$ uncertain, $4=$ disagree, $5=$ totally disagree

* Students, PhD students, researchers and teachers in healthcare

**Other relevant actors in healthcare 
Table 4 Mean, median and SD of RAS-5 score in the categories for all background variables

\begin{tabular}{|c|c|c|c|c|c|}
\hline & & Mean & Median & SD & $P$ values \\
\hline RAS-5 $(n=264)$ & & 2.38 & 2.36 & 0.64 & \\
\hline \multirow{5}{*}{$\begin{array}{l}\text { Participant } \\
\text { group }\end{array}$} & Patients & $2.59 *$ & 2.6 & 1.78 & \multirow[t]{5}{*}{$0.002 * * *$} \\
\hline & Relatives & 2.24 & 2.27 & 1.76 & \\
\hline & $\begin{array}{l}\text { Care profes- } \\
\text { sionals }\end{array}$ & 2.36 & 2.45 & 0.74 & \\
\hline & $\begin{array}{l}\text { School } \\
\text { actors } * * * *\end{array}$ & 2.52 & 2.54 & 0.64 & \\
\hline & Other***** & 2.2 & 2.21 & 0.61 & \\
\hline \multirow[t]{2}{*}{ Gender } & Woman & 2.39 & 2.36 & 0.42 & \multirow[t]{2}{*}{$0.052 * *$} \\
\hline & Man & 2.33 & 2.36 & 1.07 & \\
\hline \multirow{8}{*}{$\begin{array}{l}\text { Age (in dec- } \\
\text { ades) }\end{array}$} & $15-19$ & 2.36 & 2.54 & 0.98 & \multirow[t]{8}{*}{$0.053 * * *$} \\
\hline & $20-29$ & 2.5 & 2.45 & 0.61 & \\
\hline & $30-39$ & 2.59 & 2.54 & 0.58 & \\
\hline & $40-49$ & 2.31 & 2.27 & 0.58 & \\
\hline & $50-59$ & 2.31 & 2.27 & 0.67 & \\
\hline & $60-69$ & 2.2 & 2.18 & 0.56 & \\
\hline & $70-79$ & 2.39 & 2.52 & 0.72 & \\
\hline & $80-89$ & 1.75 & 1.41 & 1.03 & \\
\hline \multirow{3}{*}{$\begin{array}{l}\text { Mother lan- } \\
\text { guage }\end{array}$} & Swedish & 2.32 & 2.36 & 0.55 & \multirow[t]{3}{*}{$0.050 * * *$} \\
\hline & Finnish & 2.48 & 2.45 & 0.58 & \\
\hline & Other & 2.32 & 2.36 & 0.65 & \\
\hline \multirow{2}{*}{$\begin{array}{l}\text { Educational } \\
\text { level }\end{array}$} & Lower & $2.58^{*}$ & 2.54 & 0.81 & \multirow[t]{2}{*}{$0.004 * *$} \\
\hline & Higher & 2.29 & 2.27 & 0.43 & \\
\hline \multirow[t]{2}{*}{ Read/heard } & Yes & 2.35 & 2.36 & 0.41 & \multirow[t]{2}{*}{$0.074 * *$} \\
\hline & No & 2.56 & 2.59 & 1.13 & \\
\hline \multirow[t]{2}{*}{ Have met } & Yes & $2.18 *$ & 2.27 & 0.87 & \multirow[t]{2}{*}{$0.023 * *$} \\
\hline & No & 2.43 & 2.45 & 0.43 & \\
\hline
\end{tabular}

$S D$ Standard deviation

$* p<0.05$

** $T$ test with two independent samples

****One-way ANOVA with three or more independent samples

****Students, PhD students, researchers and teachers in healthcare

$* * * * *$ Other relevant actors in healthcare

$p=0.001)$ compared to patients and those with a higher education compared to those with a lower education $(r=-0.181, p=0.004)$ had statistically significant correlation. This indicates that other relevant actors in healthcare and those with a higher educational level were more likely to have positive attitudes toward humanoid robots.

The association of the RAS-5 score with the following background variables was additionally tested in regression analysis: Participant group, Age, Gender, Mother language, Educational level, Read/heard, Have met. Multiple linear regression models were created, where background variables predicted the RAS-5 score, presented in Table 5. A significant association was found in Model $1(\mathrm{~F}(11,244)=3.749$, $p=0.000)$ when all variables were included, with an adjusted $R^{2}$ of 0.106 . In Model 2, the variable with the highest P-value was removed from the model in a stepwise manner, until only those variables with $\mathrm{P}<0.05$ remained in the model (Tabachnik and Fidell 2019). A statistically significant regression model fit was also found in Model $2(\mathrm{~F}(4$, $253)=8.268, p=0.000$ ), with an adjusted $R^{2}$ of 0.102 . For the background variable Age, the RAS-5 value decreased 0.067 for each increase of decade. For Other, the RAS- 5 value was 0.190 lower compared to patients. For those with a higher educational level, the RAS-5 value was 0.293 lower compared to those with a lower educational level. For Mother language, the RAS-5 value for those with Finnish as their mother language was 0.240 higher compared to those with Swedish as their mother language.

\section{Discussion}

We undertook a survey with the aim to describe attitudes toward the use of humanoid robots in healthcare. Our main finding was that most of the participants had mostly positive attitudes toward the use of humanoid robots in healthcare. In addition, other relevant actors in healthcare compared to patients, participants with a higher educational level compared to participants with a lower educational level and older adults compared to younger adults were more likely to have a positive attitude toward the use of humanoid robots in healthcare. Through our analysis and determination of the associations between background variables and attitudes among patients, relatives, care professionals, school actors and other relevant actors in healthcare, we add further knowledge to previous research on the subject (Pripfl et al. 2016; Papadopoulus et al. 2018). As mentioned previously, given the lack of previous research on the topic, the distinctive characteristic of this study is its specific focus on humanoid robots.

We found that the humanoid robot used in our study, Pepper, was perceived to be both friendly and interesting. In accordance with an earlier study (Piezzo and Suzuki 2017), Pepper's "cute" appearance might have positively influenced the participants during the one-time interaction seen here. We even found that most of the participants had more positive attitudes toward the use of humanoid robots in healthcare than neutral or negative attitudes. This is in line with a Eurobarometer report (2012), in which the majority of European Union citizens were found to have a positive view of robots. Nevertheless, we note that as part of the study underlying the Eurobarometer report (2012) participants were only shown pictures of robots; they did not engage with actual robots. Naneva et al. (2020) revealed that face-to-face interaction with a robot might generate more positive feelings toward both the robot one was interacting with and robots in general. The participants in this study also interacted faceto-face with a humanoid robot, and our findings support that 
Table 5 Linear regression analysis results of RAS-5 as an outcome variable and background variables as independent variables

\begin{tabular}{|c|c|c|c|}
\hline & & Model $1\left(\operatorname{adj} R^{2 *}\right)$ & Model $2\left(\operatorname{adj} R^{2 * *}\right)$ \\
\hline \multicolumn{4}{|l|}{ Participant group } \\
\hline Patients & Reference & 1 & 1 \\
\hline Relatives & vs. patients & $-0.480(-0.852$ to -0.107$) * * *$ & \\
\hline Care professionals & vs. patients & $-0.287(-0.604$ to 0.030$)$ & \\
\hline School actors $* * * *$ & vs. patients & $-0.224(-0.552$ to 0.105$)$ & \\
\hline Other***** & vs. patients & $-0.445(-0.747 \text { to }-0.144)^{* * *}$ & $-0.190(-0.358 \text { to }-0.022)^{* * *}$ \\
\hline Gender & Women vs. men & $-0.022(-0.229$ to 0.186$)$ & \\
\hline Age (in decades) & vs. a decade younger & $-0.064(-0.122$ to -0.007$) * * *$ & $-0.067(-0.117$ to -0.017$) * * *$ \\
\hline \multicolumn{4}{|l|}{ Mother language } \\
\hline Swedish & Reference & 1 & 1 \\
\hline Finnish & vs. Swedish & $0.187(0.023 \text { to } 0.352)^{*}$ & $0.240(0.082$ to 0.397$) * * *$ \\
\hline Other & vs. Swedish & $-0.034(-0.368$ to 0.301$)$ & \\
\hline Educational level & Higher vs. lower & $-0.223(-0.400$ to -0.046$) * * *$ & $-0.293(-0.358$ to -0.022$) * * *$ \\
\hline Read/heard & Yes vs. no & $-0.081(-0.293$ to 0.131$)$ & \\
\hline Have met & Yes vs. no & $-0.139(-0.350-0.072)$ & \\
\hline
\end{tabular}

Coefficients, their $95 \% \mathrm{CI}$ and statistical significance are presented for two models

*In Model 1 adjusted $R^{2}$ of .106, all background variables were included

**In Model 2 adjusted $R^{2}$ of .102, only those background variables with a significant $p$ value were included. $C I$ confidence interval for B

$* * * p<0.05$

$* * * *$ Students, $\mathrm{PhD}$ students, researchers and teachers in healthcare

******ther relevant actors in healthcare

Age was an ordered scale variable with all eight classes a decade in size, except the first class which was 15-19 years. Consequently, in this analysis, we treated age as a quantitative variable, with a unit of one decade

they experienced the interaction as being positive. Heerink (2011) also highlighted that previous experience with a robot can correlate with a positive attitude toward the use of robots. Most of the participants in our study had read or heard about humanoid robots before, but only a few had met a humanoid robot previously. We maintain that our findings may be considered of interest, because of the physical (not theoretical) participant-humanoid robot interaction that formed the basis of our investigation. Despite the variation in the length of time spent on interaction seen in this study, our findings might be useful for developers in their further development of humanoid robot technology in healthcare.

Other relevant actors in healthcare, such as service personnel or politicians, had a 0.190 lower mean value in the RAS-5, indicating that compared to patients they are more likely to have a positive attitude toward the use of humanoid robots in healthcare. Even relatives had a 0.445 lower mean value in the RAS-5, indicating that compared to patients they also were more likely to have a positive attitude, although this was only significant in Model 1 . These findings are not consistent with earlier research (Broadbent et al. 2012), in which patients were seen to be more likely than care professionals to have a positive attitude toward healthcare robots. We note, however, that the participants in Broadbent et al.'s (2012) study had not physically met a robot before evaluation. To facilitate the successful implementation of humanoid robots into healthcare, a focus on patients' mixed attitudes toward robots in healthcare should be investigated in future research, including how attitudes may change following regular physical contact. We even discern from our study findings that when implementing humanoid robots in healthcare vulnerable patient groups would appear to be an unsuitable first target group. In line with an earlier study (Kuo et al. 2009), we saw that the participants in this study who had a higher educational level, had a 0.293 lower mean value in the RAS-5, indicating that they are more likely to have a positive attitude toward the use of humanoid robots in healthcare than those with a lower educational level. Thus, those areas of healthcare where higher educational levels among personnel are more common might be a more suitable first target group when introducing humanoid robots. A careful evaluation of which patient groups can be considered suitable target groups for future studies as well as which tasks are appropriate for humanoid robots to perform in healthcare are needed. In addition, more research is needed on what influences other relevant actors' positive attitudes toward the use of humanoid robots in healthcare.

Other researchers (Haring et al. 2014) have found that cultural differences might play a considerable role in influencing attitudes toward robots. We found significant 
differences between participants with different mother languages; in this study those whose mother language is Finnish had a 0.240 higher mean value in the RAS-5, indicating that they are more likely to have a negative attitude toward the use of humanoid robots in healthcare. Further studies focusing on the reasons underlying such cultural differences might be helpful when developing and implementing humanoid robots in healthcare. We even saw differences in attitude between age classes. The age classes in this study were organized by decade with the exception of the first age class (15-19 years), and we saw that with each decade increase in age class the RAS- 5 showed a 0.067 lower mean value. This indicates that older adults are more likely to have a positive attitude toward the use of humanoid robots in healthcare than younger adults. These findings somewhat contradict the findings in earlier studies; for example Kuo et al. (2009) showed that younger adults are more likely to accept social assistive robots for healthcare tasks than older adults. However, the age variable seen in this study was not completely linear, and our univariate analysis showed that the two youngest age classes (15-19 and 20-29 years) had slightly lower RAS-5 scores than those in the 30-39 age class. Such mixed findings might be linked to the functionality of the humanoid robot used in this study and the different lengths of time the participants spent interacting with it. Comparing our study's findings with earlier research without awareness of such differences could lead to misconstrued assumptions. In further studies, there should be a focus on testing humanoid robots for more specific healthcare functionalities, in specific care situations and with different age groups.

In an attempt to seek a better understanding of the impact of increasing workload and costs in healthcare (Dahl and Boulos 2013), we even recruited persons visiting or working in the hospital setting, so as to include a general perspective in the study. Still, the background variables we collected should not alone be considered decisive in explaining the differences in attitudes; other factors should also be considered, such as how receptive a person is to new things. Developed by John et al. (2008), the Big Five personality traits scale can be used to investigate openness, conscientiousness, extraversion, agreeableness and neuroticism, and could be used as a complement to the RAS-5. One could argue that an investigation of the acceptance of technology and digital competence would have been advantageous or even the inclusion of questions about humanoid robots' function or use in specific care situations. Nevertheless, we saw that the differences in attitudes were quite small. By exploring attitudes specific to humanoid robots' appearance, further understanding of what influences their use may be gained. To realize successful implementation, human attitudes and preconceptions should be taken into account when humanoid robots are developed for healthcare. By highlighting the importance of understanding attitudes toward a specific robot technology, such as humanoid robots, our study might contribute to greater knowledge on attitudes toward the development of robot technologies.

\section{Conclusion}

The Pepper humanoid robot used in our study was considered to be both interesting and friendly. Most of the participants had a mainly positive attitude toward the use of humanoid robots in healthcare. We found that other relevant actors in healthcare, those with a higher educational level and older adults were more likely to have a positive attitude. However, more research is needed on the reasons underlying negative attitudes, because such attitudes may affect the introduction of humanoid robots into healthcare. Especially patients' negative attitudes should be investigated during further robotic research. A careful evaluation of suitable first target groups as well as appropriate tasks for humanoid robots in healthcare to perform are needed.

\section{Limitations}

The study findings should be interpreted with a certain caution. The study material was randomly collected and the participants volunteered, so the findings might not be generalizable to a wider population. A weakness is that participants with more positive attitudes might have been more likely to participate. Even though we saw no tendency for the participants to automatically select a score of 3 when encountering the 1-5 scale, the RAS- 5 should be validity tested for psychometric testing in a future study. Another limitation is that the humanoid robot in this study was not tested in any real-life care situations (i.e., bathing or reminding of medication). It can also be discussed whether the usage scenario where the humanoid robot interacted with participants is related to healthcare or not. However, even if the humanoid robot engaged in activities not typical for a healthcare context, e.g., answered basic questions, danced or sang, the study sample, context and study measurement are still relevant to healthcare. It could have been beneficial to include more patients and relatives as participants, but in comparison to other studies we maintain that this study's broad sample is an advantage. All of the analysis methods we used yielded rather similar results: univariate analyses, pairwise correlations between dichotomous RAS-5 and background variables and both multiple regression models. Even the RAS-5 and age, with both variables as dichotomous or continuous, produced rather similar results. Such consistency across all modes of analyses suggests that our results are method-independent. 
Acknowledgements The authors confirm that the manuscript has not been pre-accepted or pre-published elsewhere and is not under consideration on any non-commercial or academic site.

Author contributions All authors have contributed to the preparation and drafting of the manuscript. MA, LN, HE and LF planned the design of the study. MA and LN collected the data. MA and AR analyzed the data. All authors have given final approval of the version to be published.

Funding Open access funding provided by Abo Akademi University (ABO). This work was supported by the Åbo Akademi University Foundation.

Data availability The datasets used and analyzed during the current study are available from the corresponding author.

Code availability Not applicable.

\section{Declarations}

Conflict of interest The authors report no conflict of interest.

Open Access This article is licensed under a Creative Commons Attribution 4.0 International License, which permits use, sharing, adaptation, distribution and reproduction in any medium or format, as long as you give appropriate credit to the original author(s) and the source, provide a link to the Creative Commons licence, and indicate if changes were made. The images or other third party material in this article are included in the article's Creative Commons licence, unless indicated otherwise in a credit line to the material. If material is not included in the article's Creative Commons licence and your intended use is not permitted by statutory regulation or exceeds the permitted use, you will need to obtain permission directly from the copyright holder. To view a copy of this licence, visit http://creativecommons.org/licenses/by/4.0/.

\section{References}

Alaid A, Zhou L (2014) The determinants of home healthcare robots adoption: an empirical investigation. Int J Med Inform. https://doi. org/10.1016/j.ijmedinf.2014.07.003

Azeta J, Bolu C, Abioye AA, Festus O (2018) A review on humanoid robotics in healthcare. MATEC Web Conferences 153(5):1-5. https://doi.org/10.1051/matecconf/201815302004

Baer M, Tilliette MA, Jeleff A, Ozguler A, Loeb T (2014) Assisting older people: from robots to drones. Gerontechnology 13(1):5758. https://doi.org/10.4017/2014.13.1.012.00

Beaton D, Bombardier C, Guillemin F, Bosi Ferraz M (2000) Guidelines for the process of cross-cultural adaptation of self-report measures. Spine 25(24):3186-3191

Broadbent E, Tamagawa R, Kerse N, Knock B, Patience A, MacDonald, B (2009) Retirement home staff and residents' preferences for healthcare robots. The 18th IEEEE International Symposium on Robot and Human Interactive Communication, pp 645-650. Toyama, Japan

Broadbent E, Kuo IH, Lee YI, Rabindran J, Kerse N, Stafford R, Macdonald B (2010) Attitudes and reactions to a healthcare robot. Telemedicine and E-Health 16(5):608-613

Broadbent E, Kerse N, Day K, Macdonald BA (2012) Attitudes towards health-care robots in a retirement village. Australas J Ageing 31(2):115-120
Brunda RI, Keri V, Tp S, Bhoi D (2020) Re-purposing humanoid robots for patient care in COVID-19 pandemic. Int J Health Plan Manage. https://doi.org/10.1002/hpm.3052

Dahl TS, Boulos MNK (2013) Robots in health and social care: a complementary technology to home care and tele healthcare. Robotics 3(1):1-21

Doering N, Richter K, Gross H-M, Schroeter C, Mueller S, Volkhardt M, Scheidig S, Debes K (2015) Robotic companions for older people: a case study in the wild. Annu Rev Cyberther Telemed 13(219):147-152. https://doi.org/10.3233/978-1-61499-595-1-147

Eurobarometer (2012) Public attitudes towards robots. Gesis database. https://doi.org/10.4232/1.12265

Finnish ethical delegation (2002) Ethical principles. http://www.tenk.fi/ sv/etikpr\%C3\%B6vning-inom-humanvetenskaperna/etiska-princ iper. Accessed 15 Aug 2020

Giusti L, Marti P (2006) Interpretative dynamics in human robot interaction. The 15th IEEE international symposium on robot and human interactive communication, 2006. ROMAN 2006. https:// doi.org/10.1109/ROMAN.2006.314403

Haring KS, Mougenot C, Ono F, Watanabe K (2014) Cultural differences in perception and attitude towards robots. Int $\mathrm{J}$ Affective Eng 13(3):149-157. https://doi.org/10.5057/ijae.13.149

Heerink M (2011) Exploring the influence of age, gender, education and computer experience on robot acceptance by older adults. In The sixth ACM/IEEE international conference on human-robot interaction, pp 147-148. Lausanne. https://doi.org/10.1145/19576 56.1957704

Ishiguro K, Majima Y, Sakata N (2016) Deployment of ARCS Model and Utilization of Communication Robot in Patient Education. In: Proceedings of the 9th International Joint Conference on Biomedical Engineering Systems and Technologies, 5:371-376. https:// doi.org/10.5220/0005774603710376

Ito HT, Miyagawa M, Kuwamura Y, Yasuhara Y, Tanioka T, Locsin RC (2015) Professional nurses' attitudes towards the introduction of humanoid nursing robots (HNRs) in health care settings. J Nurs Health Sci 15:73-81. https://doi.org/10.20849/ijsn.v3

John OP, Naumann LR, Soto CJ (2008) Paradigm shift to the integrative big five trait taxonomy: history, measurement, and conceptual issues. In: John OP, Robins RW, Pervin LA (eds) Handbook of personality: theory and research. The Guilford Press, New York

Kuo IH, Rabindran JM, Broadbent E, Lee YI, Kerse N, Stafford RMQ, MacDonald B.A. (2009) Age and gender factors in user acceptance of healthcare robots. In Symposium on Robot and Human Interactive Communication, Toyama, Japan, The 18th IEEE Int. Symposium on Robot and Human Interactive Communication, pp 214-219. Toyama, Japan

Kyrarini M, Lygerakis F, Rajavenkatanarayanan A, Sevastopoulos C, Nambiappan HR, Chaitanya KK, Babu AR, Mathew J, Makedon F (2021) A survey of robots in healthcare. Technologies 9(1):8. https://doi.org/10.3390/technologies9010008

McGee-Lennon MR, Wolters MK, Brewster S (2011) Usercentered Multimodal reminders for Assistive living. Proceedings of the SIGCHI Conference on Human Factors in Computing Systems (CHI), pp 2105-2114. https://doi.org/10.1145/1978942.1979248

Naneva S, Sarda Gou M, Webb TL, Prescott TJ (2020) A systematic review of attitudes, anxiety, acceptance and trust towards social robots. Int J Soc Robot 12:1179-1201. https://doi.org/10.1007/ s12369-020-00659-4

Nomura T, Sugimoto K, Syrdal D S, Dautenhahn K (2012) Social acceptance of humanoid robots in Japan: A survey for development of the Frankenstein syndrome questionnaire. In Proceedings of the12th IEEE-RAS International Conference on Humanoid Robots (Humanoids' 12). IEEE, 242-247

Nomura T, Syrdal D S, Dautenhahn, K (2015) Differences on social acceptance of humanoid robots between Japan and the UK. In Procs 4th Int Symposium on New Frontiers in Human-Robot 
Interaction. The Society for the Study of Artificial Intelligence and the Simulation of Behaviour (AISB)

Pallant J (2011) SPSS survival manual. 4th edition. A step by step guide to data analysis using Spss. Allen\&Unwin

Papadopoulus I, Koulouglioti C, Ali S (2018) Views of nurses and other health ans social care workers on the use of assistive humanoid and animal-like robots in health and social care: a scoping review. Contemp Nurse 54(4-5):425-442

Piezzo C, Suzuki K (2017) Feasibility study of a socially assistive humanoid robot for guiding elderly individuals during walking. Future Internet 9(3):30. https://doi.org/10.3390/fi9030030

Pripfl J, Körtner T, Bathko-Klein D, Hebesberger D, Weniner M, Gisinger C, Frennert S, Eftring H, Antona M, Adami I, Weiss A, Bajones M, Vincze M (2016) Result of a real world trial with a mobile social service robot for older adults. The Eleventh ACM/ IEEE International Conference on Human Robot Interaction (pp. 497-498). Christchurch, New Zealand: 2016 11th ACM/IEEE International Conference on Human-Robot Interaction (HRI). https://doi.org/10.1109/HRI.2016.7451824

Softbanks Robotics (2017) "Who is Pepper?". https://www.ald.softb ankrobotics.com/en/cool-robots/pepper
Sperber AD (2004) Translation and validation of study instruments for cross-cultural research. Gastroenterology 126(1):24-128. https:// doi.org/10.1053/j.gastro.2003.10.016

Stafford RQ, MacDonald BA, Li X, Broadbent B (2014) Older people's prior robot attitudes influence evaluations of a conversational robot. Int J Soc Robot 6:281-297

Tabachnik BG, Fidell LS (2019) Using multivariate statistics, 7th edn. Pearson, Boston

Winfield A (2012) Robotics: a very short introduction. OUP, Oxford, p 23

Zsiga K, Edelmayer G, Rumeau P, Péter O, Tóth A, Fazekas G (2013) Home care robot for socially supporting the elderly: Focus group studies in three European countries to screen user attitudes and requirements. Int J Rehabil Res 36(4):375-378. https://doi.org/10. 1097/MRR.0b013e3283643d26

Publisher's Note Springer Nature remains neutral with regard to jurisdictional claims in published maps and institutional affiliations. 